\title{
Influence of cellulose polymorphs on the polypropylene crystallization
}

\author{
Slawomir Borysiak
}

Received: 15 October 2012/ Accepted: 25 February 2013/Published online: 27 March 2013

(C) The Author(s) 2013. This article is published with open access at Springerlink.com

\begin{abstract}
Results of the hitherto research work on alkalisation of lignocellulosic materials have been much divergent. In view of the above, the subject of this study is mercerization of cellulose from pine wood. This choice of the subject permitted observation of transformation of cellulose I to cellulose II with no participation of other components of lignocellulosic materials. According to $\mathrm{X}$-ray results, during mercerization the isolated cellulose was easily (completely) transformed into cellulose II variety, while the pine wood was converted more slowly to cellulose II polymorphs. Therefore, it could be concluded that the presence of lignin and hemicelluloses in wood prevented the transformation from cellulose I to II. The main objective of this research was to establish the effect of cellulose varieties on the nucleation ability of different fillers by using differential scanning calorimetry (DSC) and polarizing microscopy. The nucleating effect of the fillers occurs only in the presence of cellulose I variety. In contrast, the presence of cellulose II variety seems to practically eliminate the nucleating effect of the fillers. Moreover, nucleation of the mercerized wood (mixture of cellulose I and II) can be also observed, but this effect is not strong. It should be emphasised that as yet no correlation has been reported between the quantitative composition of cellulose polymorphic forms (appearing not only in wood, but in cellulose isolated from wood as well) and the nucleation ability of lignocellulosic fillers.
\end{abstract}

S. Borysiak $(\bowtie)$

Institute of Technology and Chemical Engineering,

Poznan University of Technology, Sklodowskiej-Curie 1,

60-965 Poznan, Poland

e-mail: Slawomir.Borysiak@ put.poznan.pl
Keywords Polypropylene - Wood Cellulose · Polymorphs $\cdot$ Nucleation

\section{Introduction}

Composites of polypropylene with wood have been recently given much attention at research and industry centres. It has been projected that production of such composites may soon become an important sector in plastic industry, at a level of $18 \%$ in a year in North America and $14 \%$ a year in Europe [1, 2]. The interest in these materials stems from interesting and specific properties of wood used as a filler of plastics [3-6]. Wood can be an alternative to the other commonly used fillers such as carbon fibres, glass fibres, silica, talk, and others. It should be emphasised that a rational approach to designing process of production of composite materials of lignocellulose materials and polymers needs analysis of such problems as thermal stability of the natural components in the process, adhesion of the components, and nucleating ability of the components.

As follows from analysis of literature, the key problem in producing composite materials is a good interphase adhesion between the polymer matrix and the lignocellulose filler. Poor adhesion means that on processing of the composites the unmodified lignocellulose components show a tendency toward aggregation as a consequence of the intermolecular interactions between the filler molecules [7]. For this reason many authors have been searching for methods of chemical or physical modifications of the components [8-11]. Analysis of literature data has revealed many controversies; often the use of the same modifications of the lignocellulose component would bring different results. An example of such controversies is the opinions regarding the use of mercerization. The necessity of 
mercerization as a step preceding chemical modification follows from the fact that the hydroxyl groups of native cellulose are not sufficiently reactive with the modifiers applied [12]. It should be added that mercerization is also responsible for the formation of another polymorphic variety of cellulose (cellulose II), which has been described in [13-19]. Mercerization can have a beneficial effect on mechanical properties of polymer composites [20-29]. This fact has been explained by increased roughness of the lignocellulose filler as a result of removal of low-molecular components and fibrillation of the filler. Another reason behind the improvement of composites properties is the removal of lignin, which is responsible for filler agglomeration and inhibition of cellulose molecules diffusion to the chains of the polymer matrix. In general the beneficial role of mercerization is not only to remove fats and impurities from the surface of lignocellulose filler, but also to remove lignin and hemicellulose, which improves the interactions between the filler and the matrix and leads to a better dispersion of the filler in the matrix. Nevertheless, some authors have not confirmed the positive role of mercerization in improvement of macroscopic properties of the composites [30-32]. These authors studied composites of polypropylene with wood showing poor adhesion and their morphology investigation revealed numerous cracks and pores in the interphase surface.

Another problem of concern in this area is the influence of mercerization process on the polymorphism of cellulose component [13-19]. In particular, it is interesting to establish the effect of changes in the polymorphic varieties of cellulose induced by wood mercerization on interphase structure in composite materials. The filler surface can have many active sites that can be potentially responsible for high density nucleation leading to the growth of polymer crystallites in the direction perpendicular to the filler surface. This phenomenon leads to formation of a columnlike crystalline layer known as the transcrystalline layer (TCL). Transcrystallization is possible if the energetic conditions of the process of nucleation are more favorable on the filler surface than in the bulk polymer [33]. Transcrystallization has been studied for over half of a century and it has been shown that many factors affect formation of TCL structures. Although the formation of transcrystalline structures has important effect on the properties and character of the composites produced, the mechanisms of their formation have not been fully recognized and the influence of the chemical treatment of the lignocellulose component on the development of TCL structures is ambiguous and controversial. An important problem in the context of determination of the mechanisms of TCL structures formation can be the polymorphism of cellulose contained in the natural fillers. That is why the process of mercerization responsible for the appearance of cellulose polymorphic forms deserves close analysis. According to literature data, the treatment of lignocellulose materials with alkaline chemical leads to definite deterioration of the ability to form TCL structures and often inhibits their formation [34]. High nucleation activity of only one polymorphic variety of cellulose (cellulose I) can be a consequence of the match between the crystallographic structures of cellulose and polypropylene, as studied in detail by Quillin et al. [35]. On the basis of the Wittman and Lotz theory [36], Quillin et al. have found that there is a very good match between the sizes of cellulose I chains $(0.82 \mathrm{~nm})$ and methyl groups of polypropylene $(0.84 \mathrm{~nm})$. Such a match of these sizes at the molecular level can be a reason for epitaxial growth of TCL structures on the surface of cellulose I. The lattice parameters of elementary cell of cellulose II are similar to those of cellulose I but in II the cellulose chains are not in the planar configuration as they are in I. An explanation of TCL formation based on this match has been proposed by Felix et al. [37]. According to these authors, transcrystallization is a result of interactions between polypropylene and cellulose at the molecular level. The specific configuration permits the interactions between methyl groups of polypropylene and the glycoside bonds on the cellulose surface. The electron density around the methyl group is sufficiently high to enable van der Waals interactions with oxygen in the glycoside bond. The distance between two methyl along the direction $c$ is $0.65 \mathrm{~nm}$, which is close to that between the oxygen atoms in the glycoside bond of $0.66 \mathrm{~nm}$. This matching sizes can be responsible for initiation of development of transcrystalline structures.

The present work is a follow-up of our earlier studies $[38,39]$ on the mercerization of wood. In a preliminary study, we showed that the mercerization of these samples led to a decrease in the nucleating ability of the polymer matrix. The effectiveness of nucleation of the polymer matrix to a considerable degree depends on the polymorphic form of cellulose in the lignocellulose filler. Mercerization of pine wood in the conditions in which polymorphic transition of cellulose does not take place, [38], stimulates much greater nucleation activity of the filler than in the wood subjected to alkalization favoring formation of cellulose II [39]. According to DSC results, the composites with cellulose II containing filler show very low nucleating ability, similar to that of unmodified polypropylene. On the basis of earlier studies [40] whose results were interpreted in the terms of the theory of heterogeneous nucleation [41], the composites with wood containing cellulose I only (unmodified or alkalized in the conditions not inducing conversion from cellulose I to II) were found to be characterized by nucleation of epitaxial character only. In the systems with wood containing cellulose I and cellulose II, a tendency to non-epitaxial nucleation was noted. 
So far, most investigations dealing with mercerization of cellulose have only focused on whole cellulose fillers, i.e., flax, hemp, sisal, jute, and wood. The main goal of this research was to learn the effect of alkalization of cellulose (separated from wood samples) on nucleation ability of polypropylene matrix, whose effect has not been studied so far. An understanding of the relationships between polymorphic varieties of cellulose and development of supermolecular structure of polymer matrix in composite system is important for scientific progress. Moreover, knowledge of the phenomena taking place at the interphase boundary polymer/filler permits optimization of the processing leading to products of target properties.

\section{Experimental}

\section{Materials}

\section{Pine wood}

An unmodified wood (Pinus silvestris) particles, taken after sieve analysis from 1-mm mesh supplied by ForestryKaminska, Poland was used in the performed experiments.

The chemical composition of wood was as follows: cellulose content was $51.4 \%$, lignin content was $26.2 \%$, and extractive content was $3.4 \%$.

The content of lignin and extractive substances in pine wood studied was determined by TAPPI method, according to the norm PN-92/P-50092 [42]. At first the wood sample is subjected to two-stage extraction by $96 \%$ ethyl alcohol in a Soxhlet apparatus and by a mixture of ethyl alcohol and benzene at the mass ratio of 1:2. After washing in ethyl alcohol, the wood is washed in hot water and then dried at room temperature. Then the sample of a known moisture content is placed in a $72 \%$ sulphuric acid solution cooled to $10-15^{\circ} \mathrm{C}$ for $2 \mathrm{~h}$. The sample obtained is diluted with water and boiled under reflux for $4 \mathrm{~h}$. When lignin is precipitated on the bottom of the flask, the mixture is filtered and the deposit is washed with hot water till disappearance of acidic $\mathrm{pH}$. The precipitate is dried at $105{ }^{\circ} \mathrm{C}$.

The content of extractive substances is determined in a Soxhlet apparatus with dichloromethane and $96 \%$ ethyl alcohol as a solvent. The process of extraction lasts for 4-6 h, then the solvent is evaporated into the extractor and the extract is dried to constant mass at $105^{\circ} \mathrm{C}$.

\section{Cellulose}

The isolation of cellulose from pine wood was performed by the use of the Kürschner-Hoffer method [43].
The wood sample of the known moisture content is heated in a flask with a reflux condenser in a mixture of ethyl alcohol and nitric acid for $1 \mathrm{~h}$. After this time the liquid is decanted and the wood sample is flooded again with a mixture of ethyl alcohol and nitric acid and heated for $1 \mathrm{~h}$. Then the liquid is decanted and the precipitate is washed a few times with hot water, then it is flooded with hot water and heated under reflux for $30 \mathrm{~min}$. At the last stage the precipitate is filtered off and washed with distilled water until neutral $\mathrm{pH}$ followed by drying at $105{ }^{\circ} \mathrm{C}$.

\section{Polypropylene}

The isotactic polypropylene Malen F-401 was supplied by Basell Orlen Polyolefins (Plock, Poland). The melting temperature of the PP is $163-164{ }^{\circ} \mathrm{C}$. The melt flow index was about $2.4-3.2 \mathrm{~g} / 10 \mathrm{~min}$ (at $230{ }^{\circ} \mathrm{C}$ and $2.16 \mathrm{~kg}$ ) and isotacticity was $95 \%$.

\section{Mercerization process of cellulose}

The pine wood and cellulose isolated form wood were mercerized by subjecting them to $16 \% \mathrm{NaOH}$ solution treatment at room temperature for $60 \mathrm{~min}$ with vigorous stirring. The obtained slurry was filtered and thoroughly washed with distilled water until wash water reached a neutral $\mathrm{pH}$. The prepared samples were then dried at ca. $110^{\circ} \mathrm{C}$ in a vacuum oven for $48 \mathrm{~h}$ prior to further experiments.

\section{Wide angle X-ray scattering (WAXS)}

The structure of native and mercerized samples were analyzed by means of wide-angle X-ray scattering (WAXS). The diffraction pattern was recorded between 5 and $30^{\circ}$ ( $2 \theta$-angle range) in the step of $0.04^{\circ} / 3 \mathrm{~s}$. The wavelength of the $\mathrm{Cu} \mathrm{K} \alpha$ radiation source was $1.5418 \AA$, and the spectra were obtained at $30 \mathrm{~mA}$ with an accelerating voltage of $40 \mathrm{kV}$.

The deconvolution of peaks was performed by the method proposed by Hindeleh and Johnson [44], improved and programmed by Rabiej [45]. After separation of X-ray diffraction lines, the relative amount of the polymorphic phases of cellulose (cellulose I and II) can be estimated on the basis of the separated area under the peaks of cellulose I and II. The details of this calculation procedure have been introduced in earlier publication [46].

Preparation of the composites

Prior to the extrusion process, the fillers were dried until $1 \%$ moisture content in order to prevent the steaming in the extruder. The composites containing $50 \mathrm{wt} \%$ of the 
native and the mercerized samples were homogenized in Fairex extruder (Mc Neil Akron Repiquet, France) with a length-to-diameter ratio $L / D=25$. The barrel and die temperatures were $195{ }^{\circ} \mathrm{C}$ and the screw speed was $30 \mathrm{rpm}$. The material was cooled in a water bath and then pelletized. The fillers used for the experiments have an average particle size of $0.5-1 \mathrm{~mm}$. However, we would like to emphasize that shearing forces during processing leads to the partial breakage of the filler. The particle size of fillers is c.a. $0.1-1.0 \mathrm{~mm}$ [29].

\section{Morphological analysis}

The isothermal crystallization process of polypropylene in the presence wood and cellulose fillers was carried out using the hot stage optical (Linkam TP93, made in Japan) and a polarizing optical microscope Labophot-2 (Nikon, made in Japan) equipped with a Panasonic CCD camera (KR222, made in Japan). The samples were firstly heated to $210{ }^{\circ} \mathrm{C}$ and kept at this temperature for $5 \mathrm{~min}$ to erase the previous thermal effect. Finally, the samples were cooled at $20{ }^{\circ} \mathrm{C} \mathrm{min}{ }^{-1}$ to $136{ }^{\circ} \mathrm{C}$, at which isothermal crystallization of the PP was allowed. Dry nitrogen was introduced to eliminate any possible degradation during measurement.

\section{Differential scanning calorimetry (DSC)}

DSC measurements have been carried out using a Netzsch DSC 200 under nitrogen atmosphere with a volume of $30 \mathrm{ml} \mathrm{min}{ }^{-1}$. For the DSC measurements, filler particles were compounded into pellets at $50 \%$ by mass with the polypropylene. The samples were melted at a heating rate of $10{ }^{\circ} \mathrm{C} \mathrm{min}$ min $^{-1}$ to $210{ }^{\circ} \mathrm{C}$ and maintained at this temperature for $5 \mathrm{~min}$ in order to eliminate any previous thermal history of the polymer. Then, the polymer was cooled from 210 to $40{ }^{\circ} \mathrm{C}$ at cooling rate of $5^{\circ} \mathrm{C} \mathrm{min}^{-1}$. This procedure was repeated two times and only second heating and cooling were used for calculation. The kinetic parameters of crystallization process of polypropylene, such as the crystal conversion and half-time of crystallization time were determined. Based on the determined values for the enthalpy of crystallization $(H)$, the extent of crystallization (crystal conversion), $\alpha$ was calculated:

$\alpha=\frac{\int_{0}^{t}(\mathrm{~d} H / \mathrm{d} t) \mathrm{d} t}{\int_{0}^{\alpha}(\mathrm{d} H / \mathrm{d} t) \mathrm{d} t}$

From the curves of $\alpha$ against time $(t)$, the half-time of crystallization $\left(t_{0.5}\right)$ was determined as time when crystal conversion was $50 \%$.

\section{Results and discussion}

\section{WAXS diffraction analysis}

The WAXS patterns of the wood, cellulose (selected form wood) and cellulose treated with $\mathrm{NaOH}$ solution are shown in Fig. 1.

It can be found that the diffraction patterns of the wood and cellulose exhibits three peaks at $2 \Theta=15^{\circ}, 17^{\circ}$, and $22.7^{\circ}$ assigned to cellulose I. Cellulose treated with a sodium hydroxide show peaks at $2 \theta$ equal ca. $12.5^{\circ}, 20^{\circ}$, and $22^{\circ}$, which are assigned to cellulose II. The calculated results show that the native cellulose isolated from wood (cellulose I) was completely converted into cellulose II and that no cellulose I remained in the sample. The authors of [46-49] confirmed that the crystal structure of cellulose I is transformed into that of cellulose II, usually upon treatment with $\mathrm{NaOH}$ in concentrations higher than $10 \%[14,25,46,50]$.

It should be emphasized, that in our earlier studies [19, 40] we observed that it is not possible to get full conversion of cellulose I to cellulose II as a result of wood mercerization. Therefore, lignocellulose material after mercerization is a mixture of two polymorphic varieties of cellulose, i.e., for wood mercerized with $16 \% \mathrm{NaOH}$, the amount of cellulose I and II varieties is 60 and $40 \%$, respectively. It is necessary to point out that transformation of cellulose I to II in a wood sample cannot be complete due to the presence of additional components, like hemicellulose or lignin. The above phenomena can be explained by the fact that sodium hydroxide can more easily penetrate the cellulose lattice isolated from wood (without other components) as then the effectiveness of mercerization increases.

The calculated crystallinity index values of fillers were $59 \%$ for untreated wood, $45 \%$ for mercerized wood, $72 \%$ for cellulose, and $38 \%$ for mercerized cellulose,

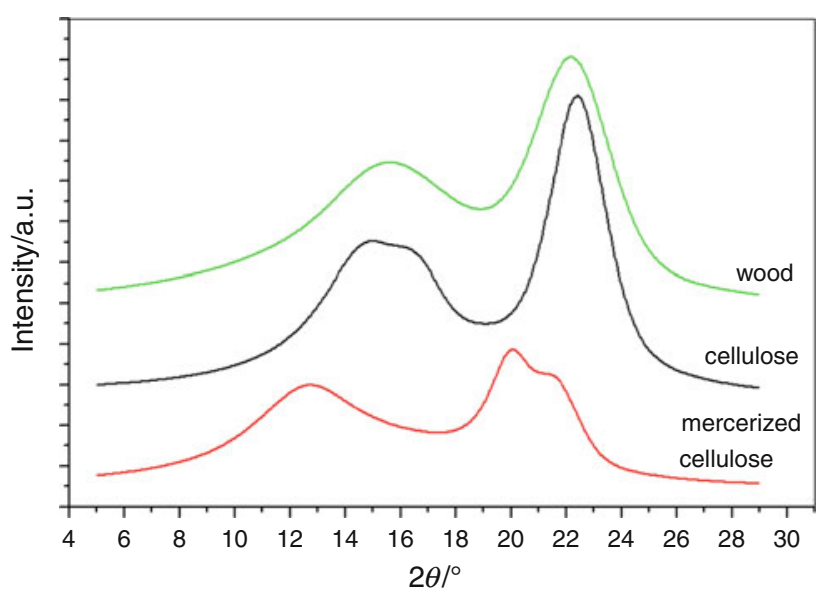

Fig. 1 WAXS patterns of wood, cellulose selected from wood and mercerized cellulose 
respectively. It can be found that the crystallinity index of fillers slightly decreases when the alkalization treatment is applied. A similar character of the dependence of cotton or ramie crystallinity during alkali treatment has been reported in recent works [51-53]. It is well known that the lignocellulosic fillers undergo three distinct process during mercerization $[18,54,55]$ : fiber swelling, disruption of the crystalline areas and formation of new crystalline lattice after rinsing away the mercerization solution. The degree of crystallinity can be well correlated with cellulose I susceptibility to transformation to cellulose II. Mercerized cellulose made of cellulose II only, is characterized by the greatest decrease in crystallinity. On the other hand, mercerization increases the disorder and changes the crystal structure as a result of cellulose I conversion to cellulose II.

Differential scanning analysis

DSC measurements can be used to investigate the kinetic parameters of polypropylene crystallization in composite systems. Figure 2 shows typical exothermic behavior of polypropylene and polypropylene-containing cellulose and wood fillers.

The peaks at $111-117^{\circ} \mathrm{C}$ are attributed to crystallization of iPP. For the pure polymer, an exothermic peak occurs at $111{ }^{\circ} \mathrm{C}$. For the composites, these exothermic peaks shift in all cases to the higher temperature. The strong increase of crystallization temperature by $6{ }^{\circ} \mathrm{C}$ was found at the iPP/ cellulose composites in comparison to pure iPP. The higher crystallization temperature values of the composites indicate that the crystallization is favored in the presence of cellulose. It is clear from Fig. 2 that the addition of mercerized cellulose to iPP caused only a marginal effect on crystallization temperature.

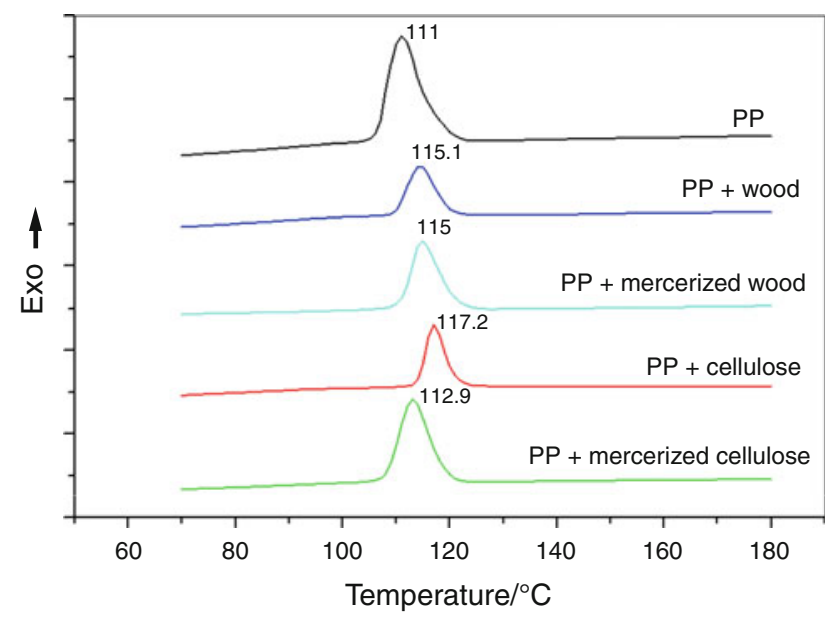

Fig. 2 DSC curves of pure PP and wood or cellulose/polypropylene composites
From DSC runs (see Fig. 2) the crystal conversion and the half-time of crystallization were determined. Figure 3 shows the variation in the crystal conversion of iPP measured in the presence of various fillers.

It is found that the crystallization of iPP is strongly influenced by the presence of a filler. All the composites show a very important increase in crystal conversion of polypropylene as compared with the pure polymer, which is attributed to a nucleant effect of fillers on iPP crystallization. However, for the composites containing cellulose II only, the decrease in the conversion of the polymer matrix is significant. Composites with mercerized cellulose are characterized by the degree of phase conversion almost identical to that of pure polypropylene. This result indicates that cellulose II is inactive of polypropylene nucleation. Similar relationships can be found for the determined values of half-times of crystallization $\left(t_{0.5}\right)$ presented in Table 1 . The $t_{0.5}$ is defined as the time necessary to reach a degree of crystalline transformation of $50 \%$.

It is evident that the iPP/cellulose and iPP/wood has a much better nucleating efficiency because of its lowest of $t_{0.5}$ value ( $\sim 1.5$ and $1.65 \mathrm{~min}$, respectively). A comparison of half-time of crystallization values evidences that the mercerized samples crystallizes longer than the iPP/cellulose and iPP/wood composites (Table 1). However, the addition of the mercerized cellulose in the polypropylene matrix no produces any modification in the crystallization of polypropylene, as can seen in Fig. 3. This suggests that the cellulose II variety (present in mercerized cellulose sample, exclusively) cancels out the nucleating effect of the filler during polypropylene crystallization. In the composites of polypropylene with mercerized wood, the presence of $40 \%$ cellulose II also produces a slight increase in $t_{0.5}$. According to decreasing $t_{0.5}$ the fillers can be ordered as follows: mercerized cellulose $>$ mercerized

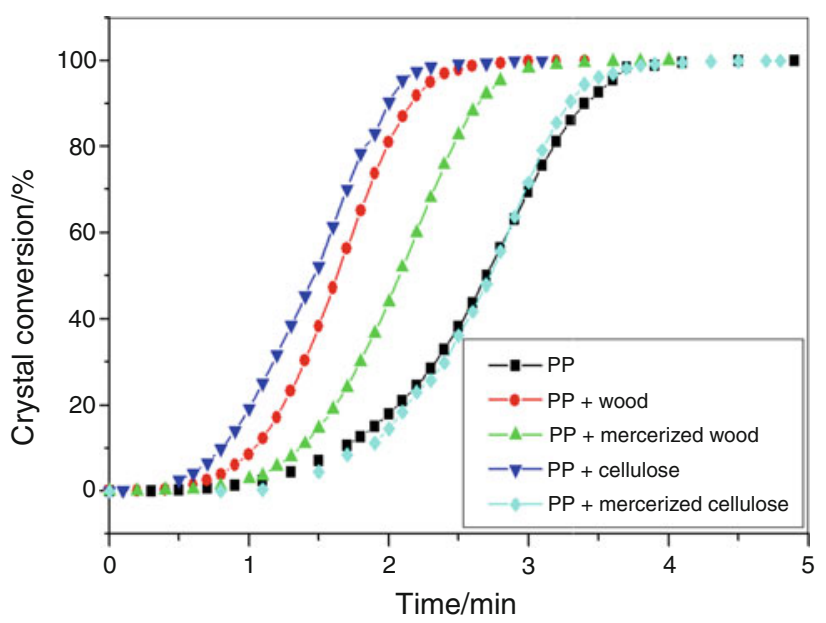

Fig. 3 The crystal conversion of pure iPP and wood or cellulose/ polypropylene composites 
Table 1 The half-time of crystallization of iPP and the composite materials

\begin{tabular}{ll}
\hline Sample & $\begin{array}{l}\text { Half-time of crystallization } \\
\left(t_{0.5}\right) / \mathrm{min}\end{array}$ \\
\hline iPP + wood & 1.65 \\
iPP + mercerized wood & 2.15 \\
iPP + cellulose & 1.5 \\
iPP + mercerized cellulose & 2.7 \\
iPP & 2.6 \\
\hline
\end{tabular}

wood $>$ wood $>$ cellulose, while the exactly opposite relationship is observed for cellulose II amount: mercerized cellulose $<$ mercerized wood $<$ wood $<$ cellulose.

As far as we are aware there are no reports in the literature of PP composites where cellulose isolated from wood and next subject to mercerization are present. From the data obtained in this work, it seems evident that the nucleating effect of the fillers occurs only in the presence of cellulose I variety. On the contrary, the presence of the cellulose II variety seems to practically eliminate the nucleating effect of the fillers. Instead, nucleation efficiency of the mercerized wood (mixture of cellulose I and II) can be also observed, but this effect is not too strong. The above phenomena can be explained by the fact, described by Bouza et al. [56], that the presence of the foreign substrate in the polypropylene reduces the critical size of the crystalline nucleus necessary for subsequent growth, since the generation of an interphase between the polymer matrix and the substrate may be less restricted than the creation of a crystalline nucleus from the melt. This means only cellulose I is effective for the formation of critical size of the crystalline nucleus and acting as an efficient-nucleating agent for the crystallization of PP.

\section{Polarizing optical microscopy}

Figure 4 shows the polypropylene crystallization process in the presence of various fillers at the temperature of $136{ }^{\circ} \mathrm{C}$.

The development of iPP transcrystallinity on the fillers is clearly shown in Fig. $4 \mathrm{a}$, c. Thanks to the nucleating ability of wood and cellulose, many nuclei are formed on the surface. The crystallization behavior of PP changes when it is mixed with mercerized wood (Fig. 4b). Alkalized wood did not induce TCL to the same extent as the untreated wood. Some nuclei at the mercerized wood surface were observed but not as many as in the unmodified wood. The SEM image of iPP mixed with mercerized cellulose (Fig. 4d) shows no transcrystalline growth. The differences between transcrystalline growths of polypropylene mixed with various fillers can be explained by analysis of the presence of cellulose varieties. The polymorphic cellulose seems to be an important factor for inducing or not the formation of transcrystalline layers. Our results suggest that heterogeneous nucleation take place during crystallization of iPP matrix when cellulose I variety is present.

These results are in agreement with those reported by Quillin and co-workers [35, 36], who have related the nucleating ability of polymorphic forms of cellulose to the
Fig. 4 Optical micrographs of polypropylene morphologies obtained in the presence of: a wood, b mercerized wood, c cellulose, $\mathbf{d}$ mercerized cellulose
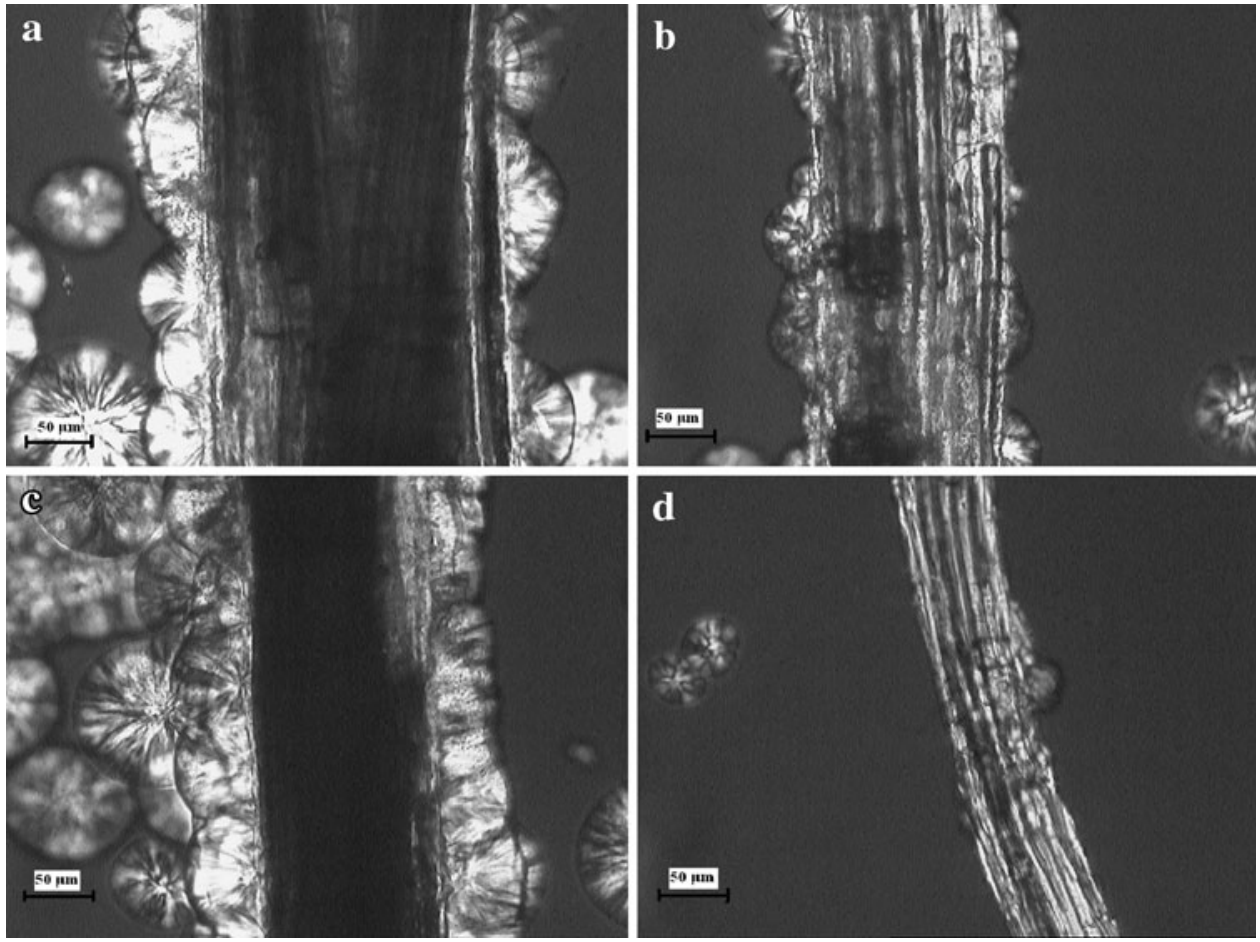
matching of crystallographic structures of cellulose and polypropylene. The size matching at a molecular level can stimulate the epitaxial growth of transcrystalline structures on the surface of cellulose I. In the earlier paper [40], we determine the type of nucleation (epitaxial or non-epitaxial) taking place upon polypropylene crystallization in the presence of the two polymorphic forms of cellulose, on the basis of the theory of heterogeneous nucleation proposed by Wunderlich [57]. Greater content of cellulose II in wood is responsible for a decrease in the nucleation activity, which is manifested by longer half-times of crystallization, lower degree of phase conversion and lower ability to form transcrystalline structures.

The influence of mercerization of lignocellulosic filler on the transcrystalline layers formation has been a subject of controversy in literature. According to some authors $[34,58]$ mercerization of the filler leads to a decrease in its nucleation abilities and eliminates transcrystallization layers, while according to others [59] it leads to development of transcrystallinity. However, it should be mentioned that the explanations proposed in this work are hypothetical with no experimental support confirming the role of polymorphic cellulose varieties (especially without a quantitative analysis).

The study presented in this paper brings a certain contribution to the actual role of polymorphic varieties in development of transcrystalline structures of polypropylene in the presence of lignocellulose fillers. It should be emphasized that as yet no correlation has been reported between the quantitative composition of cellulose polymorphic forms and the nucleation ability of the wood surface.

The polarization microscopy results corroborated those obtained by DSC investigation. It is quite evident that the presence of cellulose II variety caused a very significant reduction in the transcrystallinity layer and the crystal conversion as well as increase in half-time crystallization in comparison with the effect of cellulose I variety.

However, further consideration of this problem is necessary, and is a future problem in preparation of composite materials. In the next stage of our study, we intend to analyze the influence of such physical parameters of fillers as surface area and elemental composition/surface energy components polymer. We are convinced that the parameters presented in next our paper will contribute to better understanding a number of problem related to nucleation ability of lignocellulosic fillers.

\section{Conclusions}

Differential scanning calorimetry and polarizing optical microscopy were used to investigate the simultaneous effects of cellulose varieties and different kind of fillers on the crystallization and morphology of polypropylene matrix. The findings made from this study can shortly be summarized as follows:

- The treatment of isolated cellulose (from wood) with $16 \% \mathrm{NaOH}$ resulted in the formation of cellulose II only.

- The increase of crystallization temperature by $6{ }^{\circ} \mathrm{C}$ was found at the PP/cellulose I (selected from wood) composites in comparison to PP pure. Consequently, it can be suggested that the cellulose I act as heterogeneous nucleation agents for PP. Moreover, it is quite evident that mercerization processes of isolated cellulose caused significant reduction of the degree of the conversion phase.

- It is perfectly visible that transcrystallization is strongly influenced by kind of fillers. Mercerized cellulose did not induce the transcrystalline layer to the same extent as the treated wood with $16 \% \mathrm{NaOH}$. It is evident that the nucleation ability for PP on the untreated cellulose and wood is much higher than on the mercerized fillers.

- The results shows that the addition of filler-containing cellulose I variety (solely or partial) generates a transcrystalline phase while the filler containing cellulose II (solely) do not induce transcrystalline layer.

- The nucleation ability is increasing due to the amount of the cellulose I variety.

Acknowledgments This research was supported by Grant of Poznan University of Technology 32-171/13-DS.

Open Access This article is distributed under the terms of the Creative Commons Attribution License which permits any use, distribution, and reproduction in any medium, provided the original author(s) and the source are credited.

\section{References}

1. Wang YC, Wong PMH, Kodur V. An experimental study of the mechanical properties of fibre reinforced polymer (FRP) and steel reinforcing bars at elevated temperatures. Compos Struct. 2007;80:131-40.

2. Ashori A. Wood-plastic composites as promising green-composites for automotive industries. Bioresour Technol. 2008;99:4661-7.

3. Migneault S, Koubaa A, Erchiqui F, Chaala A, Englund K, Wolcott MP. Effects of processing method and fiber size on the structure and properties of wood-plastic composites. Compos A. 2009:40:80-5.

4. Oksman K, Mathew AP, Langstrom R, Nystrom B, Joseph K. The influence of fibre microstructure on fibre breakage and mechanical properties of natural fibre reinforced polypropylene. Compos Sci Technol. 2009;69:1847-53.

5. Faruk O, Bledzki AK, Fink HP, Sain M. Biocomposites reinforced with natural fibers: 2000-2010. Prog Polym Sci. 2012;11: 1552-96. 
6. Yang HS, Gardnem DJ, Nader JW. Dispersion evaluation of microcrystalline cellulose/cellulose nanofibril polypropylene composites using thermogravimetric analysis. J Therm Anal Calorim. 2011;103:1007-15.

7. Oksman K, Clemons C. Mechanical properties and morphology of impact modified polypropylene-wood flour composites. J Appl Polym Sci. 1998;67:1503-13.

8. Hill CAS. Wood modification: chemical, thermal and other processes. Chichester: Wiley; 2006.

9. Rowell RM. Solid wood processing: chemical modification. In: Burley J, Evans J, Youngquist J (eds) Encyclopedia of forest sciences. Oxford: Elsevier academic press; 2004. p. 1269-74.

10. Pracella M, Chionna D, Anguillesi I, Kulinski Z, Piórkowska E. Functionalization, compatibilization and properties of polypropylene composites with hemp fibres. Compos Sci Technol. 2006; 66:2218-30.

11. Danyadi L, Moczo J, Pukanszky B. Effect of various surface modifications of wood flour on the properties of PP/wood composites. Compos Part A. 2010;41:199-206.

12. Hon DNS, Ou NH J. Thermoplasticization of wood. I. Benzylation of wood. J Polym Sci Polym Chem. 1989;27:2457-82.

13. Hori R, Wada M. The thermal expansion of cellulose II and III crystals. Cellulose. 2006;13:281-90.

14. Dinand E, Vignon M, Chanzy H, Heux L. Mercerization of primary wall cellulose and its implication for the conversion of cellulose I $\rightarrow$ cellulose II. Cellulose. 2002;9:7-18.

15. Kim NH. An investigation of mercerization in decayed oak wood by a white rot fungus (Lentinula edodes). J Wood Sci. 2005;51:290-4.

16. Yue Y, Zhou C, French AD, Xia G, Han G, Wang Q, Wu Q. Comparative properties of cellulose nano-crystals from native and mercerized cotton fibers. Cellulose. 2012;19:1173-87.

17. Ibrahim M. Preparation of cellulose and cellulose derivate azo compounds. Cellulose. 2002;9:337-49.

18. Liu Y, Hu H. X-ray diffraction study of bamboo fibers treated with NaOH. Fibers Polym. 2008;9:735-9.

19. Borysiak S, Doczekalska B. X-ray diffraction study of pine wood treated with NaOH. Fibres Text East Eur. 2005;13:87-9.

20. Kuruvilla J, Sabu T, Pavithran C. Effect of chemical treatment on the tensile properties of short sisal fibre-reinforced polyethylene composites. Polymer. 1996;37:5139-49.

21. Joseph PV, Kuruvilla J, Sabu T. Effect of processing variables on the mechanical properties of sisal fiber reinforced polypropylene composite. Compos Sci Technol. 1999;59:1625-40.

22. Marconich NE, Aranguren MI, Reboredo MM. Modified wood flour as thermoset fillers. Part I. Effect of the chemical modification and percentage of filler on the mechanical properties. Polymer. 2001;42:815-25.

23. Albano C, Ichazo M, Gonzalez J, Delgado M, Poleo R. Effects of filler treatments on the mechanical and morphological behavior PP + wood flour and PP + sisal fiber. Mat Res Innovat. 2001;4: 284-93.

24. Kaith BS, Singha AS, Kumar S, Kalia S. Mercerization of flax fiber improves the mechanical properties of fiber-reinforced composites. Int J Polym Mater. 2008;57:54-72.

25. Gwon JG, Lee SY, Chun SJ, Doh GH, Kim JH. Effect of chemical treatments of wood fibers on the physical strength of polypropylene based composites. Korean J Chem Eng. 2010;27:651-7.

26. Rahman R, Islam N, Huque M. Influence of fiber treatment on the mechanical and morphological properties of sawdust reinforced polypropylene composites. J Polym Environ. 2010;18:443-50.

27. Qin C, Soykeabkaew N, Xiuyuan N, Peijs T. The effect of fibre volume fraction and mercerization on the properties of all-cellulose composites. Carbohydr Polym. 2008;71:458-67.

28. Suizu N, Uno T, Goda K, Ohgi J. Tensile and impact properties of fully green composites reinforced with mercerized ramie fibers. J Mater Sci. 2009;44:2477-82.
29. Borysiak S. Fundamental studies on lignocellulose/polypropylene composites: effects of wood treatment on the transcrystalline morphology and mechanical properties. J Appl Polym Sci. 2012; 127:1309-22.

30. Pimenta MTB, Carvalho AJF, Vilaseca F, Gironès J, Lopez JP, Mutjé P, Curvelo AAS. Soda treated Sisal/polypropylene composites. J Polym Environ. 2008;16:35-9.

31. Ichazo MN, Albano C, Gonzalez J, Perera R, Candal MV. Polypropylene/wood flour composites: treatments and properties. Compos Struct. 2001;54:207-14.

32. Raj RG, Kokta BV, Groluleau G, Daneault C. The influence of coupling agents on mechanical properties of composites containing cellulosic fillers. Polym Plat Technol Eng. 1990;29:339-53.

33. Karger-Kocsis J (editor). Polypropylene: structure, blends and composites. Londyn: Chapman \& Hall; 1995.

34. Gray DG. Polypropylene transcrystallization at the surface of cellulose fibers. Polym Lett Ed. 1974;12:509-15.

35. Quillin DT, Caulfield DF, Koutsky JA. Crystallinity in the polypropylene/cellulose system. I. Nucleation and crystalline morphology. J Appl Polym Sci. 1993;50:1187-94.

36. Wittman JC, Lotz B. Epitaxial crystallization of polymers on organic and polymeric substrates. Prog Polym Sci. 1990;15: 909-48.

37. J Felix JM, Gatenholm P. Effect of trancrystalline morphology on interfacial adhesion in cellulose/polypropylene composites. J Mater Sci. 1994;29:3043-9.

38. Borysiak S, Doczekalska B. Influence of chemical modification of wood on the crystallisation of polypropylene. Holz Roh Werkst. 2006;64:451-4.

39. Borysiak S. Determination of nucleation ability of wood for nonisothermal crystallisation of polypropylene. J Therm Anal Calorim. 2007;88:455-62.

40. Borysiak S. Influence of wood mercerization on the crystallization of polypropylene in wood/PP composites. J Therm Anal Calorim. 2012;109:595-603.

41. Muchova M, Lednicky F. Investigation of heterogeneous nucleation by the induction time of crystallization: 1 . Theory of induction time. Polymer. 1996;37:3031-6.

42. Standard: PN-92/P-50092. Raw materials for the paper industry. Wood. Chemistry analysis.

43. Kürschner K, Hoffer A. Ein neues verfahren zur bestimmung der cellulose in hölzern und. Zellstoffen Tech Chem Pap Zellst. 1929;26:125-9.

44. Hindeleh AM, Johnson DJ. The resolution of multipeak data in fibre science. J Phys Appl Phys. 1971;4:259-63.

45. Rabiej S. A comparison of two X-ray diffraction procedures for crystallinity determination. Eur Polym J. 1991;27:947-54.

46. Borysiak S, Garbarczyk J. Applying the WAXS method to estimate the supermolecular structure of cellulose fibres after mercerization. Fibres Text East Eur. 2003;11:104-6.

47. Wertz JL, Bedue O, Mercier JP. Cellulose science and technology. 1st ed. Boca Raton: Taylor and Francis Group; 2010.

48. Hon DNS. Chemical modification of lignocellulosic materials. 1st ed. New York: Marcel Dekker; 1996.

49. Ishikawa A, Okano T, Sugiyama J. Fine structure and tensile properties of ramie fibres in the crystalline form of cellulose I, II, III and IV. Polymer. 1997;38:463-8.

50. Zugenmaier P. Conformation and packing of various crystalline cellulose fibers. Prog Polym Sci. 2001;26:1341-417.

51. Flaque C, Montserrat S. Vinylic graft copolymers of cellulose. II. Thermal degradation of cellulose and its vinylic copolymers and blends by differential scanning calorimetry. J Appl Polym Sci. 1991;42:3205-13.

52. Avella M, Casale L, Dell'erba R, Focher B, Martuscelli E, Marzetti A. Broom fibers as reinforcing materials for polypropylene-based composites. J Appl Polym Sci. 1998;68:1077-89. 
53. Shiraishi N, Matsunaga T, Yokota T, Hayashi Y. Preparation of higher aliphatic acid esters of wood in an $\mathrm{N}_{2} \mathrm{O}_{4}$-DMF cellulose solvent medium. J Appl Polym Sci. 1979;24:2347-59.

54. Metshitsuka G, Idogai A. Chemical structures of cellulose, hemicelluloses, and lignin. In: Hon DNS, editor. Chemical modification of lignocellulosic materials. 1st ed. New York: Marcel Dekker; 1996.

55. Samir ASA, Alloin F, Dufresne A. Review of recent research into cellulosic whiskers, their properties and their application in nanocomposite filed. Biomacromolecules. 2005;6:612-26.

56. Bouza R, Marco C, Ellis G, Martin G, Gomez MA, Barral L. Analysis of the isothermal crystallization of polypropylene/wood flour composites. J Therm Anal Calorim. 2008;94:119-27.
57. Wunderlich B. Crystal, nucleation, growth, annealing. In: Macromolecular physics. New York: Academic Press; 1976. p. 1-70.

58. Zafeiropoulos NE, Baillie CA, Matthews FL. A study of transcrystallinity and its effect on the interface in flax fibre reinforced composite materials. Compos Part A. 2001;32:525-43.

59. Son SJ, Lee YM, Im SS. Transcrystalline morphology and mechanical properties in polypropylene composites containing cellulose treated with sodium hydroxide and cellulase. J Mater Sci. 2000;35:5767-78. 